\title{
Switchable Aperiodic Distributed Feedback Lasers: Time Domain Modelling and Experiment
}

DOI:

10.1364/CLEO_AT.2012.JW2A.101

Link to publication record in Manchester Research Explorer

\section{Citation for published version (APA):}

Chakraborty, S., Hsin, C-W., Marshall, O. P., \& Khairuzzaman, M. (2012). Switchable Aperiodic Distributed Feedback Lasers: Time Domain Modelling and Experiment. In CLEO: QELS-Fundamental Science, OSA Technical Digest (Optical Society of America, 2012), paper JW2A.101.

http://www.opticsinfobase.org/abstract.cfm?URI=QELS-2012-JW2A.101 Optical Society of America. https://doi.org/10.1364/CLEO_AT.2012.JW2A.101

\section{Published in:}

CLEO: QELS-Fundamental Science, OSA Technical Digest (Optical Society of America, 2012), paper JW2A.101. http://www.opticsinfobase.org/abstract.cfm?URI=QELS-2012-JW2A.101

\section{Citing this paper}

Please note that where the full-text provided on Manchester Research Explorer is the Author Accepted Manuscript or Proof version this may differ from the final Published version. If citing, it is advised that you check and use the publisher's definitive version.

\section{General rights}

Copyright and moral rights for the publications made accessible in the Research Explorer are retained by the authors and/or other copyright owners and it is a condition of accessing publications that users recognise and abide by the legal requirements associated with these rights.

\section{Takedown policy}

If you believe that this document breaches copyright please refer to the University of Manchester's Takedown Procedures [http://man.ac.uk/04Y6Bo] or contact uml.scholarlycommunications@manchester.ac.uk providing relevant details, so we can investigate your claim.

\section{OPEN ACCESS}




\title{
Switchable Aperiodic Distributed Feedback Lasers: Time Domain Modelling and Experiment
}

\author{
Subhasish Chakraborty, Chen-Wei Hsin, Owen P. Marshall, Md. Khairuzzaman \\ School of Electrical and Electronic Engineering, University of Manchester, Manchester, M13 9PL, UK. \\ e-mail: s.chakraborty@manchester.ac.uk
}

\begin{abstract}
We present a perturbative aperiodic distributed feedback grating embedded within a Fabry-Pérot cavity. Time domain modelling revealed electronic switching functionality, occurring only in the presence of facet reflections. The switching behaviour was experimentally verified. OCIS codes: (250.3140) Integrated optoelectronic circuits Optoelectronics; (230.0250) Optoelectronics
\end{abstract}

\section{Introduction}

Simulated annealing (SA) optimization algorithms, based on the discrete Fourier transform, are well known in diffraction optics for inverse design of computer generated holograms [1]. In this paper, we report an aperiodic distributed feedback (ADFB) cavity designed applying an SA algorithm (Fig. 1b) [2]. Unlike single-mode DFB lasers, the ADFB grating does not possess a regular or uniform periodicity in real space; instead it contains a multitude of phase-shifts with precise locations and sizes set in such a way that they operate collectively to provide a well-defined set of spatial frequency components, leading to three Bragg reflection bands centered around a frequency $f_{\mathrm{B}}$ (Figs. 1c and d), where $f_{\mathrm{B}}=c / 2 n_{\text {eff }} \Lambda$ ( $n_{\text {eff }}$ : effective refractive index, $c$ : speed of light in vacuum, $\Lambda$ : minimum grating element separation). In addition, the ADFB grating enables switching between reflection bands when integrated within a Fabry-Pérot (FP) laser cavity. To achieve this switching functionality (Fig. 1e) the individual reflections must not be too strong that frequency movement is hindered under varying laser-driving conditions, i.e. we desire only perturbative grating effect, with facet reflections remaining the primary source of feedback. Figures 1c and d illustrate the ADFB spectral complex reflection coefficient with varying arbitrary phase contributions. Lasing will occur only in the presence of unity net round-trip complex gain. The non-uniform longitudinal mode intensity within the FP-ADFB cavity produces non-linearity in the injected carrier distribution, which in turn gives rise to a non-uniform $n_{\text {eff. }}$ Therefore, with changing driving conditions, the relative optical path length between the various sub-cavities (illustrated in Fig. 1a) will change, leading to a dynamic phase relationship between sub-cavities. This effect, when combined with the ADFB complex reflectivity, leads to a bias-sensitive FP-ADFB system. We note that if the facet reflections are absent (or the grating reflections are too strong), the FP-ADFB laser does not possess the dynamic phase response (or it becomes too weak) required for mode switching. Here we present simulation results of a switchable FP-ADFB laser obtained using a time domain model (TDM), and also an experimental demonstration.

\section{Methodology}

The TDM we used divides the device into many sections and then solves the time-dependent coupled-wave equations by using a split-step transfer matrix method. Essentially, the coupled-wave equations are split into two sets of matrices: one containing the detuning factors and time derivatives and the other containing the coupling coefficient terms. For each section and time step, the forward and reverse rate equations for the optical fields are solved and electron density is updated. The ex-facet output power of the device is recorded and Fourier-transformed to produce an output spectrum [3]. Constructing the ADFB grating within the TDM involves two stages as explained in Figs. 1f-h: the first is to consider only integral multiples of the pitch; the second is to introduce the necessary phase shifts into the structure. In the TDM of conventional quarter-wave phase-shifted DFB lasers, an extra phase shift $\exp (-\mathrm{j} \phi)$ is introduced using the phase-jump matrix [3], where $\phi=\pi l / \Lambda$ and $l$ is the extra length of the pitch accounting for the additional phase delay. In our modified TDM program, multiple phase jumps are included by modifying this phase-jump matrix. The ADFB grating was focused ion beam milled [4] into a pre-characterized FP intersubband terahertz (THz) laser. In this way emission spectra could be recorded both with and without the grating in the same device, allowing its effect on the system to be clearly discerned.

\section{Results and Conclusions}

Figures 2a-d show TDM-simulated emission spectra of an FP-ADFB laser under varying electrical driving conditions, normalised with respect to $f_{\mathrm{B}}$. Switchable single-dominant-mode emission is possible originating from each of the three ADFB reflection bands, as schematically illustrated in Fig. 1e. When facet reflectivities are set to zero, neither single mode selectivity nor the switching behaviour are present under varying driving conditions, as 
demonstrated in Figs. 2e-h. Figure 2i exhibits the measured low-resolution emission spectra of a FP THz laser indicating the existence of multiple modes across a normalised spectral width of $\sim 0.044$. Figures $2 \mathrm{k}-\mathrm{p}$ show FP-ADFB spectra from the same device (powered as a single section, Fig. $2 \mathrm{j}$ ) at increasing driving currents. Two different single-dominant-modes are possible, corresponding to the two modes marked in Figs. 2c and d. The low frequency mode is not seen due to the limited gain bandwidth and its position relative to $f_{\mathrm{B}}$. In conclusion, this is the first ever TDM of an ADFB laser demonstrating switching functionality. An extension of this device concept, showing digital tunability over six Bragg reflection bands, was also recently demonstrated [5].

This work was supported by EPSRC First Grant EP/G064504/1 and partly supported by HMGCC.

(a)

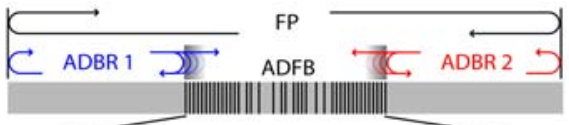

(b)

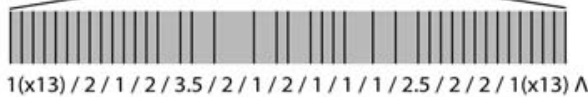

(c)

(d)

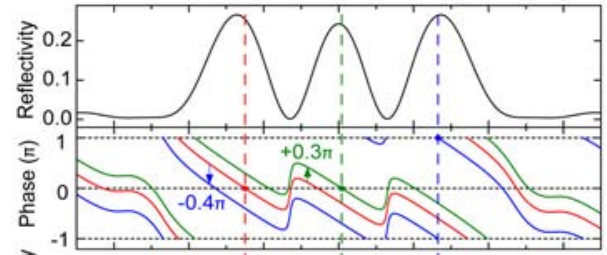

(e)

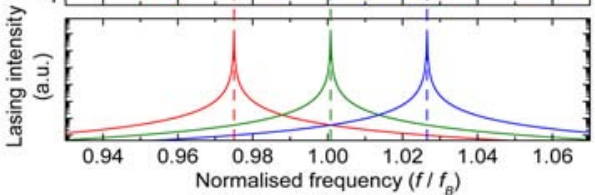

(f)

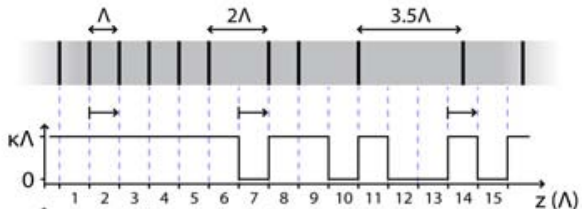

(h)

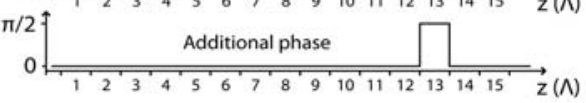

Figure 1: (a) Schematic of an FP-ADFB cavity with two resultant aperiodic distributed Bragg reflector (ADBR) sub-cavities. (b) ADFB design. Vertical lines represent narrow low refractive index slots. (c) ADFB spectral reflectivity response, calculated using ref. [2]. (d) Illustrative single-pass ADFB phase spectra $\psi(k)$ (red), with two global phase shifts (green, blue); $\psi(k)=m \pi, m=$ integer defines the possible laser oscillation frequencies. However, the round-trip complex gain of each solution is modified by Fig. 1(a), filtering out the majority of modes - for simplicity we show single-mode behaviour in (e), with switching occurring as phase varies. (f) A section of Fig. 1(b), with the corresponding distribution of (g) the coupling factor multiplied by the minimum slot separation length, and (h) the required quarter wave phase-jumps (in this section), as implemented in the TDM.
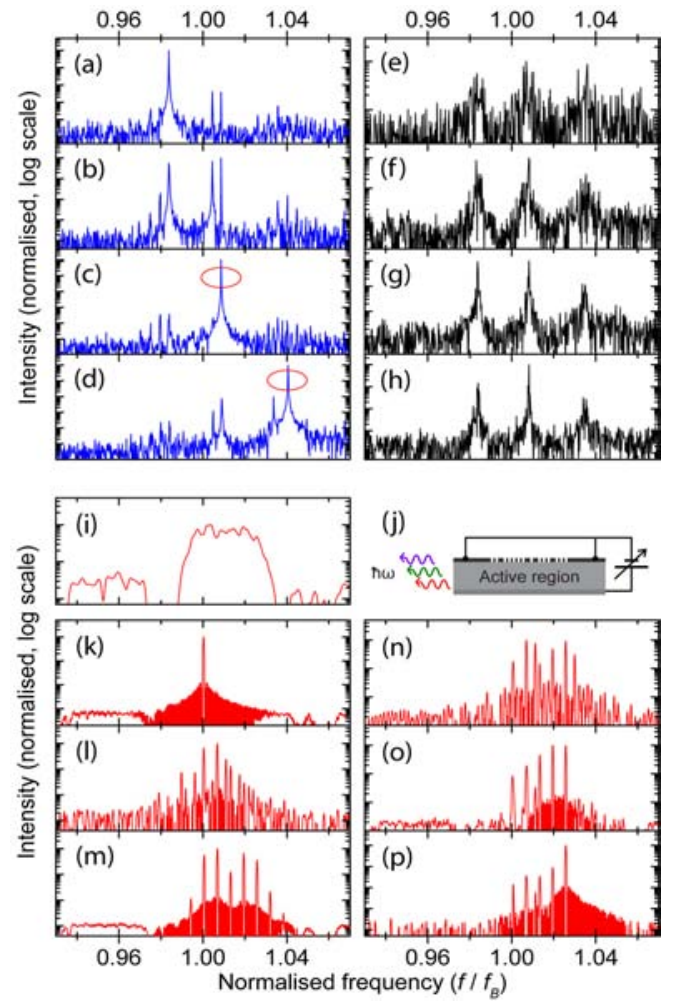

Figure 2: (a)-(h) Simulated emission spectra for different electrical driving conditions of the FP-ADFB laser, powered as a single section; (a)-(d) with facet reflections, (e)-(h) without facet reflections but with the same total device length. (i)-(p) Measured emission spectra from an intersubband $\mathrm{THz}$ laser; (i) spectra collected before ADFB introduction (FP-only), (j) FP-ADFB device schematic, (k)-(p) FP-ADFB spectra from the same device at increasing driving currents. Other FP-only spectra revealed no significant shift in gain position with increased driving current.

\section{References}

[1]T. D. Wilkinson, D. C. O’Brien, and R. J. Mears, “Dynamic asymmetric binary holograms using a ferroelectric liquid crystal spatial light modulator,” Opt. Commun. 109, 222 (1994).

[2] S. Chakraborty, M.C. Parker, and R.J.Mears, "A Fourier (k-) space design approach for controllable photonic band and localization states in aperiodic lattices,” Photon. Nanostruc. Fund. Appl. 3139 (2005).

[3] J.E. Carroll, J. Whiteaway, and R.G.S. Plumb: “Distributed Feedback Semiconductor Lasers” (IET publication, London 1998 ), Chap. 7.

[4] S. Chakraborty, et al. "Spectral engineering of terahertz quantum cascade lasers using focused ion beam etched photonic lattices," Electron. Lett. 42, 404 (2006).

[5] S. Chakraborty, O. P. Marshall, Md. Khairuzzaman, H. E. Beere and D. A. Ritchie, "Longitudinal Computer-Generated Holograms for Digitally Tunable Terahertz Lasers” - under review. 